\title{
Selected papers from SENSET2017, the first International Conference on Sensors, Networks, Smart and Emerging Technologies
}

\author{
Sawsan Sadek ${ }^{1}$ \\ Published online: 14 June 2018 \\ (C) Springer Science+Business Media, LLC, part of Springer Nature 2018
}

SENSET2017 is the first International Conference on Sensors, Networks, Smart and Emerging Technologies. It is a multi-track event that was held between 12 and 14 September 2017 in Beirut, Lebanon, a very attractive and touristic country in the Middle East.

SENSET2017 was organized by the Lebanese University, Faculty of Technology. It was technically supported by IEEE and financially supported by Lebanese University, various associations and companies who believed in this event. It was an international forum that brought researchers from different parts of the world to present the various developments, new ideas and their findings in diverse but complementary fields leading to Smart Cities.

Pr. Isam Shahrour and seven other distinguished speakers from all over the world presented their talks on Smart Cities concept and their implementation, their development, contribution to machine learning tools, competitiveness of solar energy, electrically small resonators, MIMO antenna system for $4 \mathrm{G}$ and $5 \mathrm{G}$ applications, and Electromagnetic simulation state of the art for signal processing. Furthermore, 75 accepted papers were presented and are published on the IEEE Xplore Digital Library.

By this Editorial, I would like to thank Dr. Mohammad Ismail, ALOG Editor, who offered us the opportunity to make a special issue for SENSET2017. Thus, 13 extended versions of the best presented papers in the conference are in this special issue of the Springer Journal ALOG. Various research subjects are discussed in those papers as listed below:

Sawsan Sadek

sawsansadek70@gmail.com

1 Department of Communications Computer Networks Engineering, Faculty of Technology, Lebanese University, Saida, Lebanon
- From Narrow-Band to Ultra-Wide-Band Microwave Sensors in Direct Skin Contact for Breast Cancer Detection

- Smart water technology for leakage detection: feedback of large-scale experimentation

- Determinant characteristics in EEG signal based on bursts amplitude segmentation for predicting pathological outcomes of a premature newborn, with validation using ANN

- Graphene oxide-Nylon ECG sensors for wearable IoT healthcare: nanomaterial and SoC interface

- Towards Smarter City: Clever School Transportation System

- Heartbeat rate measurements using microwave systems: single-antenna, two-antennas, and modeling moving person

- Uncovering Copy-Move Traces using Principal Component Analysis, Discrete Cosine Transform and Gabor Filter

- Exploitation of the ambient noise for the structural health monitoring of bars and tubes

- A framework for enhancing mobile workflow execution through injection of flexible security controls

- Compact substrate integrated waveguide sensor for liquids permittivity measurement

- Use of the smart technology for water quality control: feedback from large-scale experimentation

- Emotion Recognition in Arabic Speech

- A Novel Low SAR Water-Based Mobile Handset Antenna

Finally I would like to extend my thanks and appreciation to all those authors who submitted papers, to the reviewers and to all members of the organizing committee and the technical program committee for their dedication and commitment and for making SENSET 2017 a great success.

Sawsan Sadek

Saida, Lebanon, May 2018-05-17 


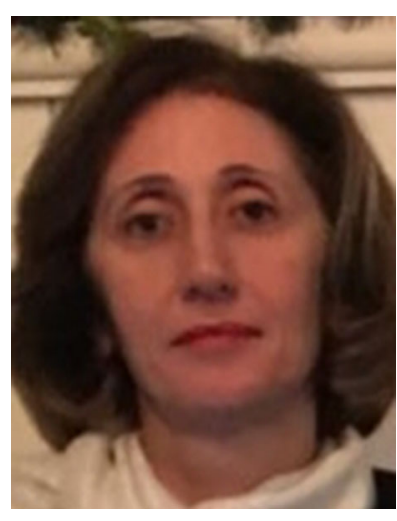

Sawsan Sadek received the B.S.

She participated to several scientific committees for international degree in physics from Lebanese University in 1989 and Ph.D. degree in Microwaves from the University of Sciences and Technologies of Lille, France, in 1996. Actually, Sawsan is a professor at the Lebanese University, Faculty of Technology in Saida, Lebanon, Department of Communications, Computer and Networks Engineering. She chaired the department for 5 years from 2009 to 2014. She organized the MMS2013 in Saida, Lebanon and SENSET2017 in Beirut, Lebanon. conferences and has more than 7 journal papers, 20 international conferences and one book chapter. 